\title{
The VMC survey - XLIV: Mapping metallicity trends in the Large Magellanic Cloud using near-infrared passbands
}

\author{
Samyaday Choudhury ${ }^{1,2 \star}$, Richard de Grijs ${ }^{1,2}$, Kenji Bekki ${ }^{3}$, Maria-Rosa L. Cioni ${ }^{4}$, Valentin D. Ivanov ${ }^{5}$, \\ Jacco Th. van Loon ${ }^{6}$, Amy E. Miller ${ }^{4,1}$, Florian Niederhofer ${ }^{4}$, Joana M. Oliveira ${ }^{6}$, Vincenzo Ripepi ${ }^{7}$, \\ Ning-Chen Sun $^{8}$ and Smitha Subramanian ${ }^{9}$ \\ ${ }^{1}$ Department of Physics and Astronomy, Macquarie University, Balaclava Road, Sydney, NSW 2109, Australia \\ 2 Research Centre for Astronomy, Astrophysics and Astrophotonics, Macquarie University, Balaclava Road, Sydney, NSW 2109, Australia \\ 3 ICRAR, M468, University of Western Australia, 35 Stirling Hwy, 6009 Crawley, Western Australia, Australia \\ 4 Leibniz-Institut für Astrophysik Potsdam (AIP), An der Sternwarte 16, D-14482 Potsdam, Germany \\ 5 European Southern Observatory, Karl-Schwarzschild-Str. 2, D-85748 Garching bei München, Germany \\ 6 Lennard-Jones Laboratories, Keele University, ST5 5BG, UK \\ 7 INAF-Osservatorio Astronomico di Capodimonte, via Moiariello 16, I-80131, Naples, Italy \\ 8 Department of Physics and Astronomy, University of Sheffield, Hicks Building, Hounsfield Road, Sheffield S3 7RH, UK \\ 9 Indian Institute of Astrophysics, II Block, Koramangala, Bengaluru 560 034, India
}

Accepted XXX. Received YYY; in original form ZZZ

\begin{abstract}
We have derived high-spatial-resolution metallicity maps covering $\sim 105 \mathrm{deg}^{2}$ across the Large Magellanic Cloud (LMC) using near-infrared passbands from the VISTA Survey of the Magellanic Clouds. We attempt to understand the metallicity distribution and gradients of the LMC up to a radius of $\sim 6 \mathrm{kpc}$. We identify red giant branch (RGB) stars in spatially distinct $Y,\left(Y-K_{\mathrm{s}}\right)$ colour-magnitude diagrams. In any of our selected subregions, the RGB slope is used as an indicator of the average metallicity, based on calibration to metallicity using spectroscopic data. The mean LMC metallicity is $[\mathrm{Fe} / \mathrm{H}]=-0.42 \mathrm{dex}(\sigma[\mathrm{Fe} / \mathrm{H}]=0.04 \mathrm{dex})$. We find the bar to be mildly metal-rich compared with the outer disc, showing evidence of a shallow gradient in metallicity $\left(-0.008 \pm 0.001 \mathrm{dex} \mathrm{kpc}^{-1}\right)$ from the galaxy's centre to a radius of $6 \mathrm{kpc}$. Our results suggest that the LMC's stellar bar is chemically similar to the bars found in large spiral galaxies. The LMC's radial metallicity gradient is asymmetric. It is metal-poor and flatter towards the southwest, in the direction of the Bridge. This hints at mixing and/or distortion of the spatial metallicity distribution, presumably caused by tidal interactions between the Magellanic Clouds.
\end{abstract}

Key words: stars: abundances - Hertzsprung-Russell and colour-magnitude diagrams - galaxies: abundanes - Local Group - Magellanic Clouds

\section{INTRODUCTION}

The Magellanic Clouds (MCs), comprising the Large Magellanic Cloud (LMC) and the Small Magellanic Cloud (SMC), are among the nearest dwarf satellite galaxies (at distances of, respectively, $\sim 50$ and $61 \mathrm{kpc}$; de Grijs et al. 2014; de Grijs \& Bono 2015). Together with the Milky Way (MW), they represent the closest example of an interacting system of galaxies (Murai \& Fujimoto 1980; Tanaka 1981; Fujimoto \& Murai 1984; Gardiner et al. 1994; Westerlund 1997). Studies using the Hubble Space Telescope (Kallivayalil et al. 2006a,b, 2013) suggest that the MCs are experiencing their first infall towards the MW. Simulations show that the origin of various dynamical features seen in the outskirts and inter-cloud regions, e.g., the Magellanic Bridge or Stream, may be caused

\footnotetext{
* E-mail: samyaday.choudhury@gmail.com
}

by periodic interactions between the Clouds (Diaz \& Bekki 2011; Besla et al. 2012; Wang et al. 2019).

The SMC exhibits dynamically distorted features in its stellar populations because of interactions with the LMC (e.g. Nidever et al. 2013; Subramanian et al. 2017; El Youssoufi et al. 2019; Niederhofer et al. 2021; Gaia Collaboration et al. 2021). Several distinct (sub)structures have been discovered in the LMC's outskirts (e.g. Mackey et al. 2016, 2018; El Youssoufi et al. 2021), some of which could have resulted from interactions with the SMC. Spatial mapping of the galaxies' metallicity distribution, including of any metallicity gradients (MGs), is an important tool to observe and understand the effects of the Clouds' evolution and interactions on their stellar chemical content. In Choudhury et al. (2020, henceforth $\mathrm{C} 20$ ), we created SMC metallicity maps out to a radius of $4^{\circ}$ from the galaxy's optical centre. We found evidence of asymmetric radial MGs in the SMC, which appeared flatter 
towards the LMC. This is a potential indicator of stellar mixing, likely caused by LMC-SMC interactions. Here, we check if similar effects are seen in the LMC.

The most accurate way to estimate metallicities is by means of spectroscopic studies. Several studies have used Ca II triplet (CaT) spectroscopy of red giant branch (RGB) stars to study star clusters and field regions in the LMC. Most studies were limited in both sample size (a few tens of clusters and a few hundred RGB stars) and spatial coverage. Either little or no evidence of radial MGs has been reported (e.g. Olszewski et al. 1991; Cole et al. 2005; Grocholski et al. 2006; Carrera et al. 2008; Pompéia et al. 2008; Lapenna et al. 2012). With recent large-area spectroscopic surveys of the MCs, e.g., the Apache Point Observatory Galactic Evolution Experiment's (APOGEE; Majewski et al. 2017) southern sky survey (Nidever et al. 2020), opportunities have opened up for studying MGs, as well as of the individual elemental abundances and chemo-kinematics in the MCs. Nidever et al. (2020) reported metallicities of 3600 RGB stars based on high-resolution $H$-band spectra from the APOGEE survey. Skowron et al. (2021) used the APOGEE data of red giant (RG) stars from Nidever et al. (2020) to estimate an LMC MG of $-0.026 \pm 0.002 \mathrm{dex}^{\mathrm{deg}^{-1}}$ out to around $8^{\circ}$.

Since the LMC occupies a wide area across the sky, large photometric surveys are helpful to estimate photometric metallicities and their variation across the galaxy. Spectroscopic surveys like APOGEE do not cover the MCs homogeneously to the same extent as large-area photometric studies. Photometric estimates, although not as accurate as spectroscopic ones, can help map relative metallicity variations across a galaxy and estimate global properties including radial MGs. Cioni (2009) estimated an LMC MG of $-0.047 \pm 0.003 \mathrm{dex} \mathrm{kpc}^{-1}$ out to $\sim 8 \mathrm{kpc}$ using asymptotic giant branch (AGB) field stars. They used the $\mathrm{C} / \mathrm{M}$ (Carbon to Oxygen) ratios of their AGB stars as a metallicity indicator. Although these authors covered a large area across the LMC, their indicators (AGB stars) and calibrators (RGB stars) were different, and the $\mathrm{C} / \mathrm{M}$ ratio is potentially susceptible to age effects. Later, Feast et al. (2010) re-analysed the data of Cioni (2009) and confirmed a small gradient out to about $4 \mathrm{kpc}$.

Choudhury et al. (2016, henceforth C16) estimated first-oftheir-kind metallicity maps for the LMC by combining largearea optical ( $V$ - and $I$-band) photometric surveys and CaT spectroscopic data of RGB stars. They estimated MGs out to a radius of $4 \mathrm{kpc}:-0.049 \pm 0.002 \mathrm{dex} \mathrm{kpc}^{-1}$ and $-0.066 \pm 0.006$ dex $\mathrm{kpc}^{-1}$ based on Magellanic Cloud Photometric Survey (MCPS; Zaritsky et al. 2004) and Optical Gravitational Lensing Experiment phase III (OGLE III; Udalski et al. 2008) data, respectively. Recently, Grady et al. (2021) used photometric data of RGB stars from Gaia data release 2 (DR2) and combined them with other photometric surveys, including the Wide-field Infrared Survey Explorer (WISE; Wright et al. 2010), the Two Micron All Sky Survey (2MASS; Skrutskie et al. 2006) and APOGEE spectroscopic data, to estimate metallicity maps of the MCs by employing machinelearning techniques. They estimated a negative LMC MG of $-0.048 \pm 0.001 \mathrm{dex} \mathrm{kpc}^{-1}$ out to $12 \mathrm{kpc}$, in agreement with Cioni (2009) and C16.

Here, we employ C16's technique to produce LMC metallicity maps using the near-infrared (NIR) VISTA Survey of the Magellanic Clouds (VMC; Cioni et al. 2011). The VMC survey covers an approximately two to three times larger area than the OGLE III and MCPS surveys. We will thus be able to derive metallicity maps and gradients out to $\sim 6.5 \mathrm{kpc}$, covering a much larger area than C16.

The layout of this paper is as follows. In Section 2 we describe the LMC VMC data. Section 3 contains our analysis of the RGB slope estimation and its calibration to metallicity. We present our main results, the NIR metallicity maps of the LMC, in Section 4. A discussion is presented in Section 5. We summarise our conclusions in Section 6 .

\section{DATA}

The VMC survey is a uniform and homogeneous survey of the Magellanic System in NIR passpands $\left(Y, J\right.$ and $\left.K_{\mathrm{s}}\right)$ using the $4 \mathrm{~m}$ VISTA telescope (Sutherland et al. 2015) at La Silla Paranal Observatory, Chile. It is one of the European Southern Observatory's (ESO) public surveys. We direct readers to Cioni et al. (2011) for a detailed description of the survey and its science goals. The VMC observations began in 2009 and were completed in 2018 . The survey covers about $\sim 170 \mathrm{deg}^{2}$ of the Magellanic System using the VISTA infrared camera (Dalton et al. 2006). The area coverage is $\sim 105 \mathrm{deg}^{2}$, of which $42 \mathrm{deg}^{2}, 20 \mathrm{deg}^{2}$ and $3 \mathrm{deg}^{2}$ cover the LMC, SMC, and the Magellanic Bridge and Magellanic Stream, respectively. The survey is deep, with total exposure times of $2400 \mathrm{~s}, 2400 \mathrm{~s}$ and $9000 \mathrm{~s}$ in the $Y, J$ and $K_{\mathrm{s}}$ passbands, respectively. A single tile of VMC observation represents a mosaic of six paw-print images in a given passband $\left(Y J K_{\mathrm{s}}\right)$. The number of such tiles covering the LMC is 68 . Each of these tiles covers almost uniformly an area of $1.5 \mathrm{deg}^{2}$ (by a minimum of two pixels; Sutherland et al. 2015) and their centres extend out to $6.5 \mathrm{kpc}$ from the LMC's centre.

In this study, we use the point spread function (PSF) photometry catalogue of the LMC. PSF photometry was performed following Rubele et al. (2015). The catalogue was calibrated using the VISTA photometric zero-points (v1.3). Similar to C20 (see their Section 2), we excluded sources with bad pixels and possible extended sources by selecting stars with sharpness values between -1 and +1 (see Rubele et al. 2015 , their appendix). Overall, the $Y$ - and $K_{\mathrm{s}}$-band photometry has a typical uncertainty of $\leq 0.15 \mathrm{mag}$, for stars brighter than $Y=19 \mathrm{mag}$ and $K_{\mathrm{s}}=18.5 \mathrm{mag}$. For our analysis we consider only the upper part of the RGB in the $\left(Y, Y-K_{\mathrm{s}}\right)$ colour-magnitude diagrams (CMDs), provided that the stars are brighter than the above magnitude limits. We only consider stars with photometric uncertainties $\leq 0.15$ mag.

\section{ANALYSIS}

\subsection{Estimation of the RGB slopes}

The LMC tiles are divided into 30 or more finer areas (referred to as subregions throughout the text) based on stellar density. We will briefly describe our method below. However, readers are directed to section 3 of $\mathrm{C} 16$ for a detailed description of the steps adopted to estimate the RGB slope in the CMDs of the subregions. Their technique identifies the dominant population and estimates its slope for field populations. A salient feature of the technique is that we adopt the densest part of the red clump (RC) in the relevant CMD as the 


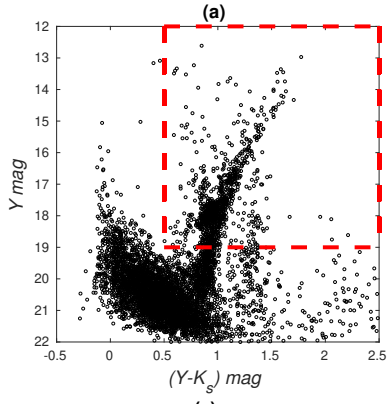

(c)

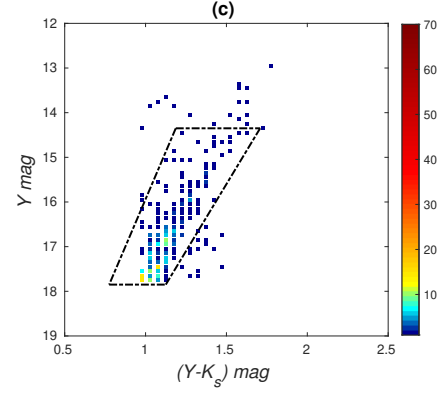

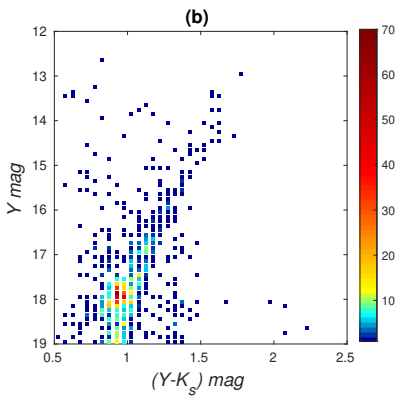

(d)

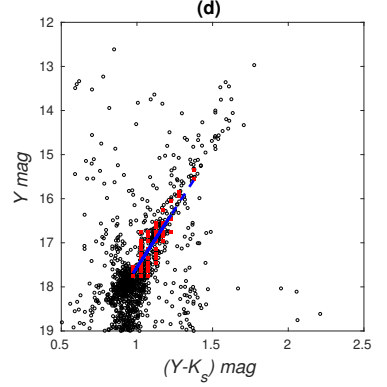

Figure 1. (a) $Y$ versus $\left(Y-K_{\mathrm{S}}\right) \mathrm{CMD}$ of a $7.85 \times 8.10 \mathrm{arcmin}^{2}$ $\mathrm{LMC}$ subregion at RA $=71.49^{\circ}, \mathrm{Dec}=-70.28^{\circ}$ in tile LMC 5_2, containing $N=6351$ stars (black points). Stars in the rectangle (red dashed line) belong to the evolved part of the CMD. (b) Density diagram of the evolved part of the CMD, where the bins are colour-coded based on stellar numbers (see the colour bar). (c) Density diagram following the application of a colour-magnitude cut at the peak of the RC distribution. The trapezoid (black dashed lines) was defined to select the RGB region and remove the dominant MW contamination. (d) CMD of the subregion (black points) overplotted with bins containing $\geq 3$ stars each (red filled squares). A linear fit to these bins representing the RGB is shown as the blue dashed line. The colour bars in (b) and (c) represent the numbers of stars in each colour-magnitude bin. The estimated parameters are: $\mid$ slope $\mid=5.27 \pm 0.48 \mathrm{mag} \mathrm{mag}^{-1}, r=0.86$ and $N_{p}=44$.

base of the RGB. Since the CMD loci of the RC and RGB stars are similarly affected by reddening, the technique can be automated for the entire LMC, irrespective of the prevailing reddening. Following C20, we selected the two extreme VMC passbands, $Y$ and $K_{\mathrm{s}}$, for our study. This affords us access to the longest colour baseline, and ensures that we have access to the maximum observable effect of metallicity on the upper RGB (the RGB segment brighter than the RC), and hence on the RGB slope (for further explanation, see Section 3 of $\mathrm{C} 20$ ). The main differences between this study and C20 are related to the removal of MW contamination.

We define the estimated parameters for the subregions consistently with respect to our previous work. The total number of stars in a subregion is denoted by $N$. We define $N_{p}$ as the number of CMD bins (with the number of stars in each bin $\geq 3$ ) representing the RGB. These bins are fitted with a straight line and the slope (|slope $\left.\mid \pm \sigma_{\text {slope }}\right)$ is estimated using least-squares minimisation (using $3 \sigma$ clipping in a single iteration). The correlation coefficient of the fit (also for other fits in the remainder of the paper) is assessed by its absolute value, $r$. We emphasise that, similarly to our previous studies, we are interested in the relative variation in RGB slope (hence in metallicity) among subregions rather than in absolute estimations for individual subregions.

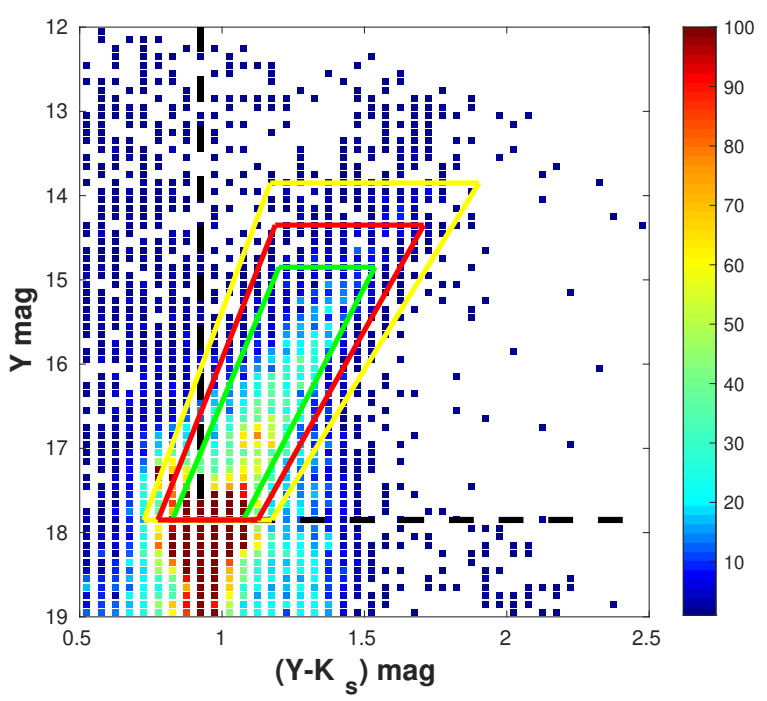

Figure 2. Density CMD for six combined subregions selected from the central LMC and from the outer parts in four different directions (see text for details). The black dashed lines show the colour-magnitude cut at the peak of the RC distribution. Three different sizes of trapezoids are shown (yellow, red and green solid lines) to select the dominant RGB population and subtract MW contamination. All are defined with respect to the RC peak. We use the red trapezoid for our analysis. The colour bars have the same meaning as those in Fig. 1.

(i) We begin by excluding the main sequence and isolating the evolved portion of the CMD (Fig. 1a). Then, we construct a density distribution of the evolved portion to identify the locus of the RC. We adopt the peak in the RC's $\left(Y-K_{\mathrm{s}}\right)$ colour and $Y$ magnitude as the base of the RGB (Fig. 1b) and apply a colour-magnitude cut at this peak (Fig. 1c).

(ii) Next, we adopt a trapezoid to select the RGB region and discard MW contamination. The MW contamination is defined by two features (see Rubele et al. 2012). The one bluer than the RGB and resembling a vertical bright strip in the vicinity of the $\mathrm{RC}$ distribution is removed by the colour and magnitude cut applied at the RC peak. The other feature is similar to a vertical strip in the redder part of the CMD, close to the RGB base. This is because the LMC is closer and and more metal-rich than the SMC. This choice of trapezoid proved to be better than the stepped block adopted in C20 to remove MW contamination. We define the trapezoid with its base anchored to the peak colour and magnitude of the RC distribution. Hence, the RGB selection criteria are consistent across all subregions.

Fig. 2 shows how we select the shape and size of the trapezoid. The shape is similar to that of region ' $\mathrm{K}$ ' adopted by El Youssoufi et al. (2019) to select the RGB population from $\operatorname{VMC}\left(K_{\mathrm{s}}, J-K_{\mathrm{s}}\right)$ CMDs to estimate morphological maps. We start by creating a density diagram by combining CMDs of subregions from different locations within the LMC: central region - tiles LMC 7_4 and 7_5; outer regions in all four directions - tiles LMC 2_5, 5_2, 6_9 and 9_3. We plot trapezoids of various sizes to sample the dominant part of the RGB population. We define the trapezoid carefully so as to avoid the redder MW contamination and avoid removing portions 

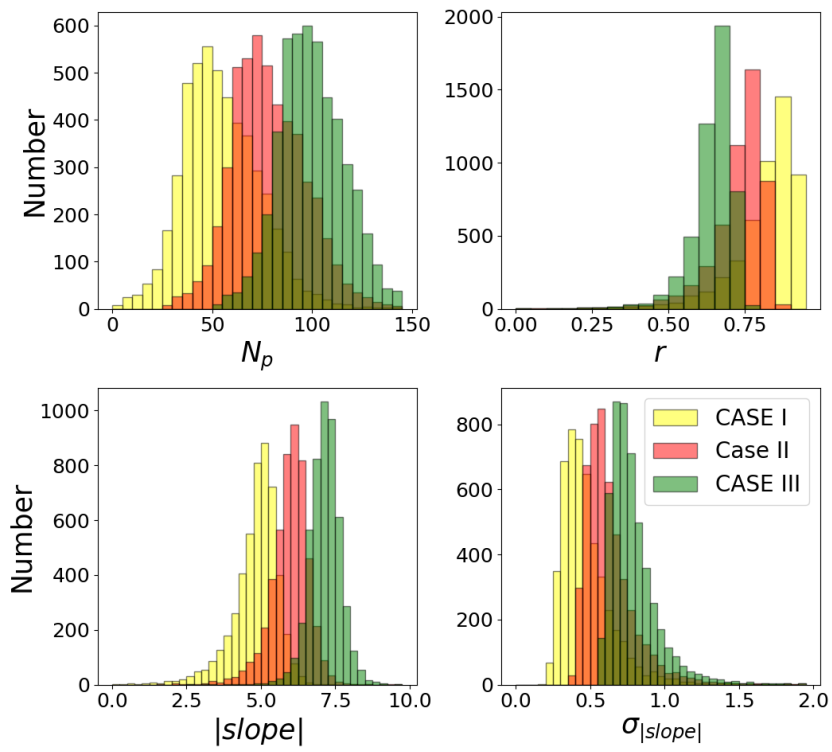

Figure 3. Histograms of parameters $\left(N_{p}, \mid\right.$ slope $\mid, \sigma_{\text {slope }}$ and $r$ ) estimated for the RGB adopting different sizes of the trapezoid considered to select the RGB region. Case II is for the final size adopted. Cases I and III are trapezoids covering smaller and larger areas, respectively, as shown in Fig. 2. The histograms are shifted with respect to each other by small constant values along the $x$ axis to avoid overlap. Offsets are $25,-0.10,1.0$ and 0.15 for $N_{p}, r$ |slope| and $\sigma_{\text {slope }}$, respectively.

of the RGB. We check for systematic effects for three choices of size on our estimated parameters: see Fig. 3. We hardly find any difference in the distributions of the estimated parameters for any of the three different sizes adopted. For our analysis, we eventually proceed with the red trapezoid shown in Fig. 2. We also verified that removing MW contamination from within the upper RGB region has a negligible effect on the resulting slope values. According to El Youssoufi et al. (2019) only 6.4 per cent of stars in the upper RGB region are from the MW. This implies that the RGB is still the dominant population there. Decontamination using Gaia proper motions may lead to slope variations of $\pm 0.05-0.15$, which is well within the $1 \sigma$ uncertainties.

Table 1 shows the nine criteria we adopted to divide our LMC tiles so as to have similar ranges of $N_{p}$ (see Fig. 4, top) for all criteria. Inspection of subregions with high stellar density suggested that small-scale variations in reddening and/or multiple dominant populations may cause the RGB to broaden, resulting in poor |slope| estimation. For regions with lower stellar density (generally the outer regions) $N_{p}$ could be low, leading to poorly defined RGBs and, hence, uncertain $r$. We thus defined 4940 subregions, with areas ranging from $13.90 \times 19.20 \operatorname{arcmin}^{2}\left(201.79 \times 278.73 \mathrm{pc}^{2}\right)$ to $3.47 \times 4.80 \operatorname{arcmin}^{2}\left(50.37 \times 69.68 \mathrm{pc}^{2}\right)$. This subdivision is necessary (see C16), since very large $N_{p}(>100)$ or small $N_{p}$ $(<20)$ leads to poor value estimations of $\mid$ slope $\mid$ with $r<0.5$. Fig. 4 (bottom) shows the resulting $N_{p}$ versus $r$ distribution.

We sample against subregions with poor slope estimations by defining four different cut-off criteria in terms of $r, \sigma_{\text {slope }}$ and $N_{p}$. Fig. 5 (top) shows a large scatter in the slopes for $N_{p}<20$, which could be artefacts owing to sparsely populated RGBs. In Fig. 5 (bottom), we note that the clumpiest
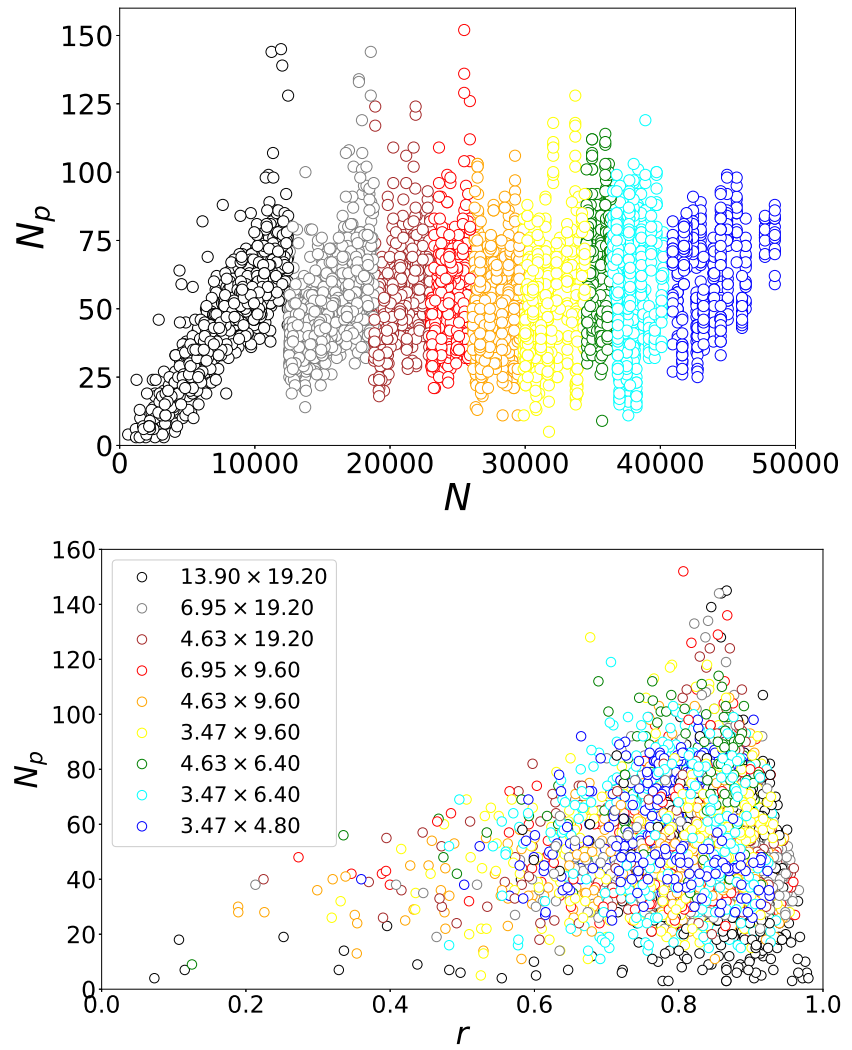

Figure 4. (Top): $N_{p}$ versus $N$ for LMC subregions following finer binning. The colours correspond to the different bin areas; see Table 1, column (8). (Bottom): corresponding $N_{p}$ versus $r$ distribution.

part of the distribution is found for $r>0.6$ and $\sigma_{\text {slope }}<1.0$. Most of the $r$ values are $>0.5$ for $\sigma_{\text {slope }}<1.5$. We observe a relatively large scatter for subregions with $r<0.5$ and $\sigma_{\text {slope }}>1.5$. Thus, our four cut-off criteria $\left(N_{p} \geq 20\right.$ for all $)$ are:

- criterion I: $r \geq 0.5$ and $\sigma_{\text {slope }} \leq 1.5$;

- criterion II: $r \geq 0.5$ and $\sigma_{\text {slope }} \leq 1.0$;

- criterion III: $r \geq 0.6$ and $\sigma_{\text {slope }} \leq 1.5$;

- criterion IV: $r \geq 0.6$ and $\sigma_{\text {slope }} \leq 1.0$.

Fig. 6 shows a comparison of the RGB slope distribution for all four cutoff criteria with respect to the original sample. The distribution is curtailed on the side with lower RGB slopes as we tighten our criteria. The peaks and overall shape of the distribution are not affected significantly as we move from criterion (I) to (IV). The numbers of subregions removed are 3.7 and 5.6 per cent for criteria (I) and (IV), respectively. Thus, even with the strictest criterion we end up with a statistically significant number of subregions (4663) to proceed with our analysis. We point out that calibrating the PSF catalogue using photometric zero-points contained in v1.5 (GonzálezFernández et al. 2018) instead of v1.3 will not affect our results, since any differences in the estimated RGB slopes will be well within the $1 \sigma$ uncertainties. 
Table 1. The nine binning criteria used to subdivide LMC tiles. For each criterion, column (2) indicates the limit on the total number of stars $(N)$ within a region. Column (3) lists the number of regions within that limit. Columns (4) and (5) specify the numbers by which regions are binned along RA and Dec, respectively. Column (6) lists the total number of subregions. Column (7) gives the area of each such subregion, and column (8) denotes the total number of subregions corresponding to each of the six subdivision criteria. The colours adjacent to the numbers are used to denote them in Fig. 4 (top and bottom).

\begin{tabular}{|c|c|c|c|c|c|c|c|}
\hline & Stars & $\begin{array}{c}\text { Regions } \\
\text { (a) }\end{array}$ & $\begin{array}{c}\text { RA } \\
\text { divisions } \\
\text { (b) }\end{array}$ & $\begin{array}{c}\text { Dec } \\
\text { divisions } \\
\text { (c) }\end{array}$ & $\begin{array}{c}\text { No. of } \\
\text { divisions } \\
(d=b \times c)\end{array}$ & $\begin{array}{c}\text { Area } \\
\left(\operatorname{arcmin}^{2}\right)\end{array}$ & $\begin{array}{l}\text { Subregions } \\
\qquad(a \times d)\end{array}$ \\
\hline 1 & $0<N \leq 12,500$ & 902 & 1 & 1 & 1 & $(13.90 \times 19.20)$ & 902 (black) \\
\hline 2 & $12,500<N \leq 18,800$ & 538 & 2 & 1 & 2 & $(6.95 \times 19.20)$ & 1076 (gray) \\
\hline 3 & $18,800<N \leq 23,000$ & 172 & 3 & 1 & 3 & $(4.63 \times 19.20)$ & 516 (brown) \\
\hline 4 & $23,000<N \leq 26,000$ & 99 & 2 & 2 & 4 & $(6.95 \times 9.60)$ & 396 (red) \\
\hline 5 & $26,000<N \leq 29,800$ & 84 & 3 & 2 & 6 & $(4.63 \times 9.60)$ & 504 (orange) \\
\hline 6 & $29,800<N \leq 34,500$ & 65 & 4 & 2 & 8 & $(3.47 \times 9.60)$ & 520 (yellow) \\
\hline 7 & $34,500<N \leq 36,500$ & 26 & 3 & 3 & 9 & $(4.63 \times 6.40)$ & 234 (green) \\
\hline 8 & $36,500<N \leq 40,500$ & 34 & 4 & 3 & 12 & $(3.47 \times 6.40)$ & 408 (cyan) \\
\hline 9 & $N>40,500$ & 24 & 4 & 4 & 16 & $(3.47 \times 4.80)$ & 384 (blue) \\
\hline
\end{tabular}

\subsection{Calibration of the RGB slope as a function of metallicity}

We use the same spectroscopic studies as C16 for calibration: metallicities of RGs in the bar of the LMC (Cole et al. 2005), star clusters (Grocholski et al. 2006) and fields covering areas $6 .^{\prime} 8 \times 6^{\prime} .8$ around these clusters (A. A. Cole, private communication). All three studies were conducted by the same group using the FORS2 instrument on ESO's $8.2 \mathrm{~m}$ Very large Telescope. The authors used the CaT lines to estimate the metallicities of their RGs, including the calibration of CaT strength to $[\mathrm{Fe} / \mathrm{H}]$. Thus, there are no inconsistencies or systematic offsets among these studies. Together, these three studies cover a range in metallicity and location across the LMC, which is important for our calibration of the RGB slopes. We use a similar technique as C16 (see their Section 3.1) to estimate the mean metallicities of the subregions and star clusters/fields around them.

Cole et al. (2005) estimated the metallicities of 373 field RGs within a $200 \operatorname{arcmin}^{2}$ area at the optical centre of the LMC bar. Their metallicity distribution is sharply peaked at the median value, $[\mathrm{Fe} / \mathrm{H}]=-0.40 \mathrm{dex}$, with a small tail of stars extending down to $[\mathrm{Fe} / \mathrm{H}]=-1.21 \mathrm{dex}$. We estimate the mean metallicity for a subregion by averaging over the Cole et al. metallicities within the relevant area. While doing so we consider stars located within twice the standard deviation about the mean metallicity. To ensure a good calibration, we consider those subregions with $r \geq 0.70, \sigma_{\text {slope }} \leq 0.5$ and which contain spectroscopic metallicity estimates for at least five RGs. These are indicated by blue points in the $[\mathrm{Fe} / \mathrm{H}]$ versus |slope| plane shown in Fig. 7.

Grocholski et al. (2006) estimated the abundances of 28 LMC clusters, aged between 1 to 13 Gyr, whose metallicities ranged between -0.30 and -2.0 dex. We use the central coordinates and radii of those lying within the VMC coverage to extract their data and construct $\left(Y, Y-K_{\mathrm{s}}\right)$ CMDs. We then employ our technique to estimate the RGB slopes for these clusters. For most of the clusters, the RGB is either sparsely populated or exhibits significant scatter about the mean RGB (either due to crowding or mixing of cluster and field stars in the VMC data or, less likely, differential reddening). For sparsely populated clusters we relax our RGB identification criteria. We select colour-magnitude bins with $N_{p} \geq 2$ stars (instead of 3 ) to recover their RGB slopes. The RGB slopes and mean $[\mathrm{Fe} / \mathrm{H}]$ values of the star clusters are shown as green points in Fig. 7. A similar analysis is carried out for the field stars, starting with their extraction from VMC fields from which the cluster area is subtracted, estimating the RGB slopes from $\left(Y, Y-K_{\mathrm{s}}\right)$ CMDs. For some of the sparse fields, we identify the RGB using a lower cut-off in colour-magnitude bins (i.e., $N_{p} \geq 2$ stars). The resulting $\mathrm{RGB}$ slopes and the mean $[\mathrm{Fe} / \mathrm{H}]$ values of these fields are shown in red in Fig. 7.

In Fig. 7 we see that the RGB slopes range from 4.25 to 6.25 , covering most of the RGB slope distribution shown in Fig. 6. The mean metallicity range covers ranges from -0.30 to $-0.80 \mathrm{dex}$. To estimate a slope-metallicity relation, we perform linear least-squares fits with two different clipping choices, $3 \sigma$ and $1 \sigma$ :

$[\mathrm{Fe} / \mathrm{H}]=(-0.08 \pm 0.04) \times \mid$ slope $\mid+(0.001 \pm 0.19) \operatorname{dex}, r=0.37$;

$[\mathrm{Fe} / \mathrm{H}]=(-0.10 \pm 0.02) \times \mid$ slope $\mid+(0.09 \pm 0.13) \mathrm{dex}, r=0.59$.

Their slopes and $y$-intercept values agree within the errors. The $1 \sigma$-clipped relation has a higher $r$ but a lower reliability of the estimated slope-metallicity relation. We use both to estimate the metallicities and compare our results in Sections 4 and 5. Our calibration of the RGB slope to metallicity rests on the assumption that the spectroscopic targets are drawn from the dominant population in the subregions. A direct comparison of the slope-metallicity relation with $\mathrm{C} 16$ is not possible since the relation is a function of the wavelengths used for these studies. We observe a scatter in the plot resulting in lower $r$ compared with that of C16 where $r \sim 0.50$ for the $3 \sigma$-clipped case. However, for this study we used twice more calibration points (36) compared with C16 (16). The total number of spectroscopic RGs used to estimate the $3 \sigma$-clipped slope-metallicity relation is 486 . We also investigated APOGEE spectroscopic data for our calibration, 

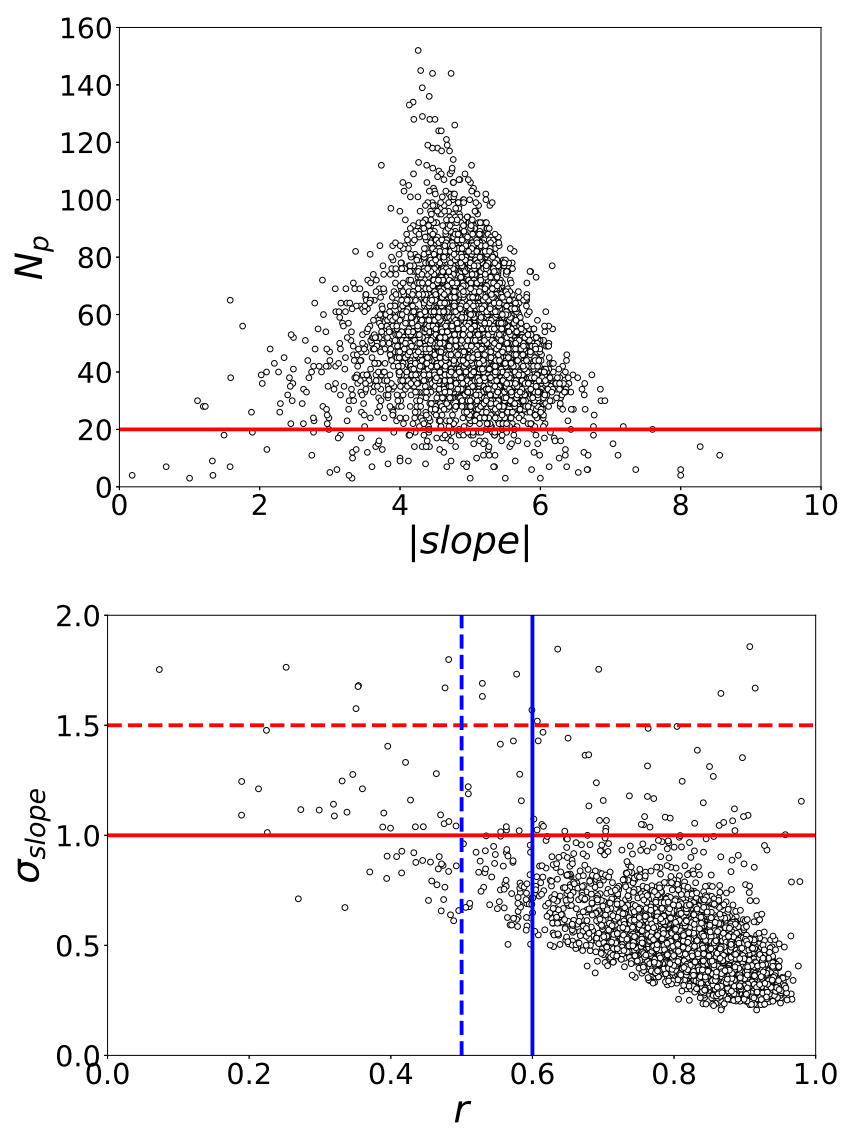

Figure 5. (Top): $N_{p}$ versus |slope| for the subregions. The red line at $N_{p}=20$ denotes the cut-off adopted to exclude regions with poorly populated RGBs. (Bottom): $\sigma_{\text {slope }}$ versus $r$ for the subregions. The red dashed and solid lines correspond to cut-off criteria for $\sigma_{\text {slope }}$ of 1.5 and 1.0, respectively. The blue dashed and solid lines denote cut-offs corresponding to $r=0.5$ and 0.6 , respectively.

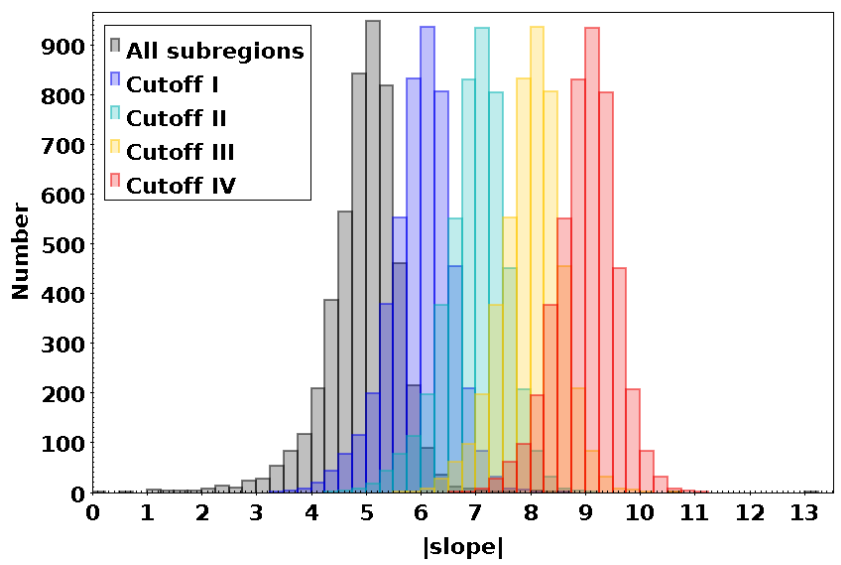

Figure 6. |slope| histogram for LMC subregions pertaining to all four cut-off criteria, compared with the equivalent distribution for no cut-off. Each histogram is shifted by 1.0 along the $x$ axis with respect to the previous histogram to avoid overlaps.

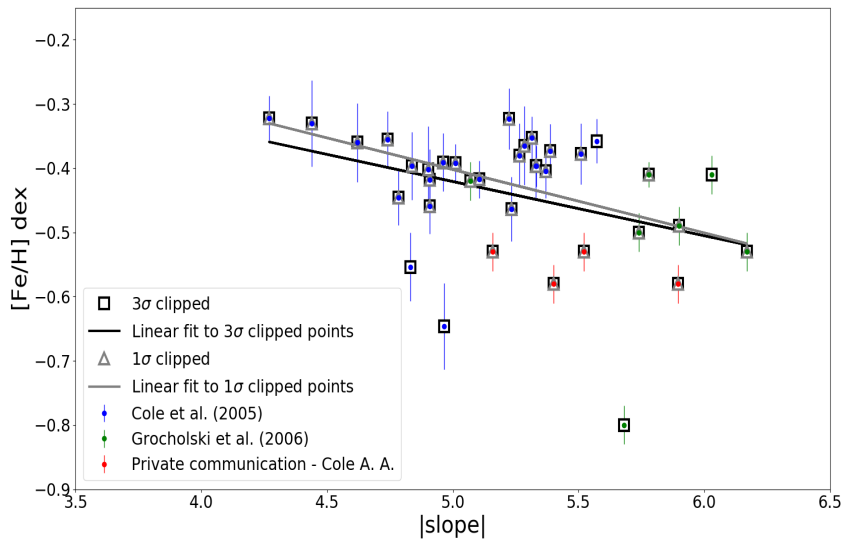

Figure 7. Metallicity $([\mathrm{Fe} / \mathrm{H}])$ versus $\mid$ slope|. Blue points denote our subregions whose mean $[\mathrm{Fe} / \mathrm{H}]$ has been found using RGs from Cole et al. (2005), green points denote clusters from Grocholski et al. (2006) and red points correspond to fields around Grocholski et al. (2006)'s clusters provided by Andrew A. Cole. The grey open squares and solid line denote the $3 \sigma$-clipped points and their corresponding best fits. The black open triangles and solid line denote the $1 \sigma$-clipped points and their corresponding best fit. The error bar (vertical coloured line) shown for each point is the standard error in the mean $[\mathrm{Fe} / \mathrm{H}]$.

but the calibrators had insufficient range in RGB slope and metallicity values, to estimate a relation.

\section{NIR METALLICITY MAPS OF THE LMC}

Fig. 8 shows the estimated metallicties for the LMC subregions in the projected sky plane using Equation (1). For the LMC's centre we adopted RA $=5^{\mathrm{h}} 19^{\mathrm{m}} 38^{\mathrm{s}}$ and Dec $=-69^{\circ}$ $27^{\prime} 5.2^{\prime \prime}$ (J2000.0 de Vaucouleurs \& Freeman 1972). The bar region shown in the figure is in accordance with Subramanian \& Subramaniam (2010). We estimated metallicity maps for all four cut-off criteria mentioned in Section 3. As we move from cut-off criterion (I) to (IV), we lose 3.7 to 5.6 per cent of subregions. For C16 the loss of subregions was 16.9 and 33.7 per cent for OGLE III and MCPS maps, respectively. Thus, we have a more or less uniform spatial distribution with smaller gaps compared with the C16 maps. We did not find any differences in global features among the four NIR maps. Hence, we present the map with the strictest cut-off criteria. The NIR metallicity map of the LMC covers an area that is about thrice larger compared with the optical maps of $\mathrm{C} 16$. The OGLE III maps covered primarily the bar region and the eastern and western parts of the LMC out to $\pm 4.0^{\circ}$ along their $x$ axes (see fig. 13 of C16). MCPS maps covered $\pm 3.5-4.0^{\circ}$ uniformly along the $x$ and $y$ axes (see fig. 29 of $\mathrm{C} 16)$. The VMC maps reveal trends out to $\pm 6^{\circ}$ along the $y$ axes and beyond and to $\pm 4.5^{\circ}$ along the $x$ axes.

The bar region is almost homogeneous with the more metal-rich points $([\mathrm{Fe} / \mathrm{H}]>-0.35 \mathrm{dex})$ located near the 30 Doradus (30 Dor) star-forming region, and some in the northwestern (NW) part of the bar. The most metal-poor regions $(<-0.55 \mathrm{dex})$ are located in the outer disc (at radii $>2^{\circ}$ ). We note a shallow variation of $[\mathrm{Fe} / \mathrm{H}]$ from the bar to the outer regions as compared with the maps of $\mathrm{C} 16$. 


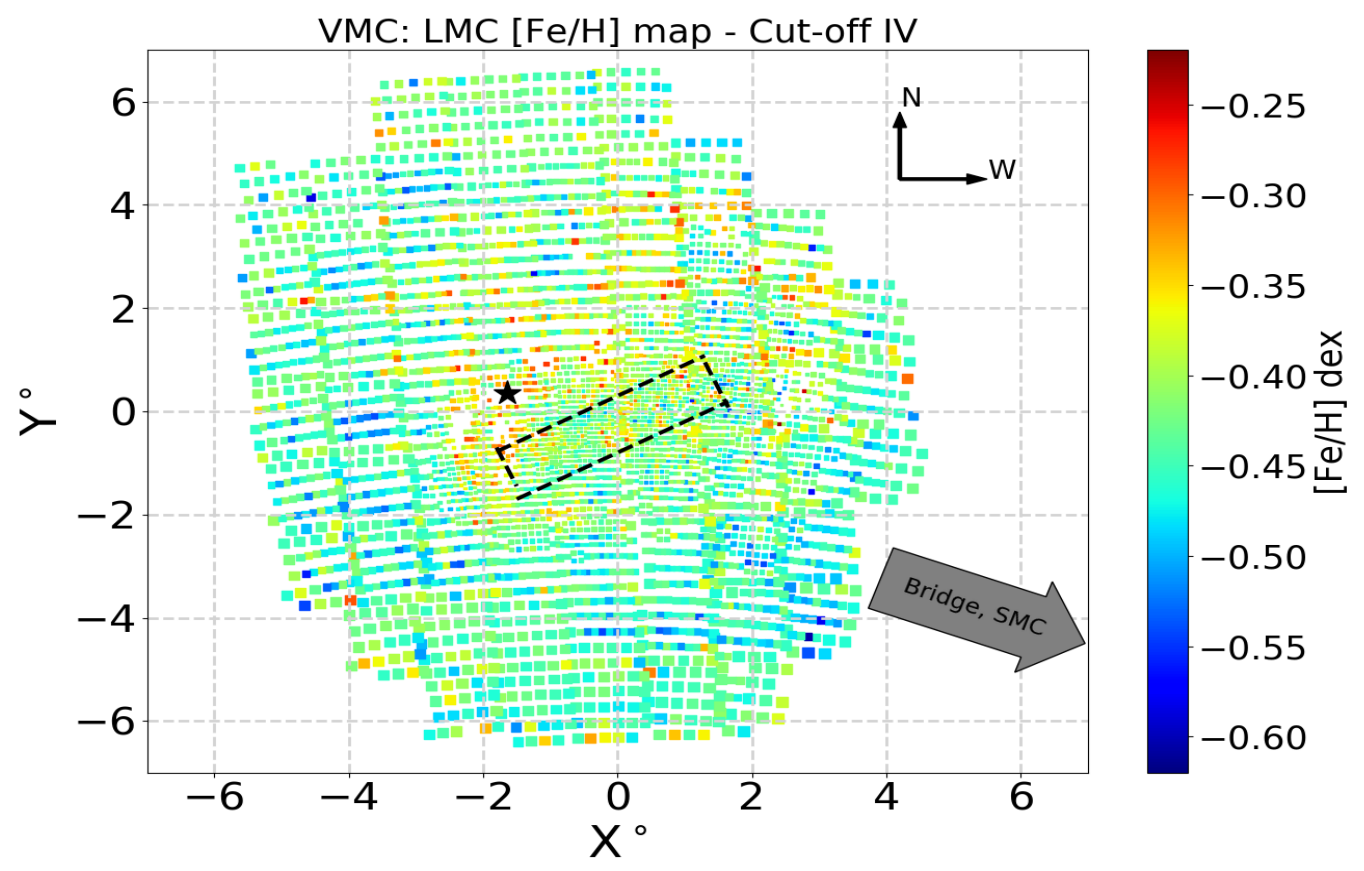

Figure 8. Metallicity map based on cut-off criterion (IV) using Equation (1). The bar region is shown by a dashed rectangle near the centre. The location of 30 Dor is shown by a black star.

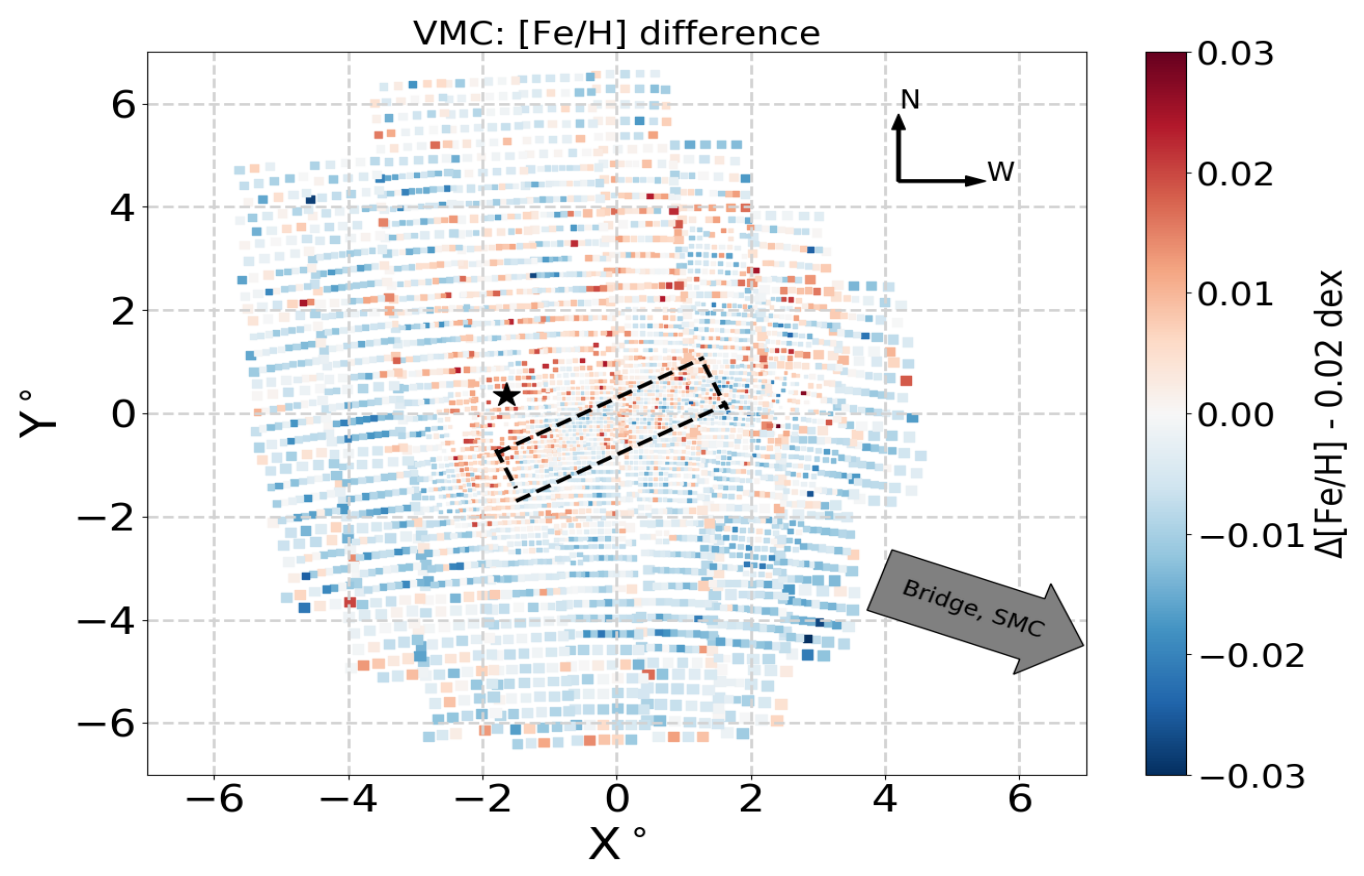

Figure 9. Difference in metallicities, $\Delta[\mathrm{Fe} / \mathrm{H}]$. The difference is defined as 'metallicities estimated using Equation (1) subtracted from the corresponding values estimated using Equation (2)'. The $\Delta[\mathrm{Fe} / \mathrm{H}]$ values are shifted by -0.02 dex to bring the zero values to the centre of the colour bar. The location of the bar and 30 Dor are shown as in Figure 8.

The metallicity in the NIR maps ranges from -0.22 dex to -0.60 dex, whereas the C16 maps showed a wider variation from -0.10 dex to -0.90 dex. We note that the northern disc of the LMC has a larger range in metallicity compared with the southern part. This is similar to what was observed in the MCPS maps of C16. The eastern and western disc of the LMC have a similar range in metallicity, a trend which is also observed in the OGLE III maps of C16. Some gaps appear in the maps; the most prominent ones correspond to the 30 Dor region and to regions North of the bar. These regions most likely suffer from issues caused by crowding or large variations in reddening. Such gaps are more prominent 
in the optical maps of $\mathrm{C} 16$, and in particular for the MCPS data set compared with OGLE III. This is most likely related to poor seeing conditions and/or the low resolution of the MCPS images. However, with the NIR maps we have been able to cover most of these gaps.

We also used Equation (2) to estimate metallicities. Fig. 9 shows the map of differences between the metallicities estimated using the two different slope-metallicity calibration relations. For all subregions, Equation (2) results in enhanced metallicities compared with Equation (1). This is owing to the fact that Equation (2) has a steeper slope and a more metal-rich $y$ intercept compared with Equation (1). The mean difference between the metallicity estimates from these two calibration relations is small, however, $0.018 \pm 0.007 \mathrm{dex}$. A byproduct of C16's work was a metallicity map of outliers, which identified significantly different metallicities in the LMC bar and outskirts. The metal-poor outliers were found in the northern, eastern and western disc, whereas the metal-rich counterparts were primarily seen in the bar region and in a few locations in the north. We were unable to estimate a map of metallicity outliers as in C16, since we recover a smaller metallicity range in this study. We checked our results by plotting subregions that deviate by $\pm 1 \sigma$ about the mean metallicity $(\sim-0.42 \mathrm{dex})$ of the LMC disc. This showed that the most metal-rich points $(>-0.31 \mathrm{dex})$ are located in the bar, with only a few metal-poor $(>-0.53$ dex $)$ points scattered in the outskirts. CaT studies of 1000 LMC field giants by Olsen et al. (2011) found that a fraction of them represented a metal-poor, kinematically distinct population in the LMC's disc - possibly accreted from the SMC. However, like $\mathrm{C} 16$, we did not find any correlation between the subregions identified as metal-poor outliers and the locations in which Olsen et al. (2011) discovered a possible accreted SMC population. This is possibly because at none of these locations the kinematically distinct population is dominant enough to turn the mean metallicity of the subregions significantly metal-poor relative to neighbouring fields. This could be because the total accreted population is either very small or well-mixed into the LMC disc as a whole.

We also compare our metallicity maps with the most recent results of Grady et al. (2021). Their LMC metallicity map extends out to $12^{\circ}$ and shows a central metal-rich bar with the most metal-poor regions littering the outskirts. The global features in their map are generally consistent with those of C16 and the present study. Also, we observe the northern and southern portions of the LMC disc to be metal-enhanced compared with the eastern and western parts, which is consistent with the results of Grady et al. (2021) and C16. Those authors also found metal-rich structures tracing the main spiral arm in the north and a prominent spiral-like feature in the southern portion of the disc. El Youssoufi et al. (2019)'s morphology map of the LMC's RGB population (their fig. 6) shows an enhanced bar feature with some diffuse structures around the bar. For younger populations ( $\lesssim 1$ Gyr old) the bar is less prominent and spiral features appear in the northern and southern regions of the disc. It is possible that our NIR metallicity maps represent a population with ages older than 1 Gyr.

We have calculated the errors associated with our metallicity estimates ( error $_{[\mathrm{Fe} / \mathrm{H}]}$ ) using error propagation following equation (4) of $\mathrm{C} 16$ (see their section 5). Fig. 10 shows that the range of error $[\mathrm{Fe} / \mathrm{H}]$ spans from $\sim 0.22$ dex to 0.34 dex,

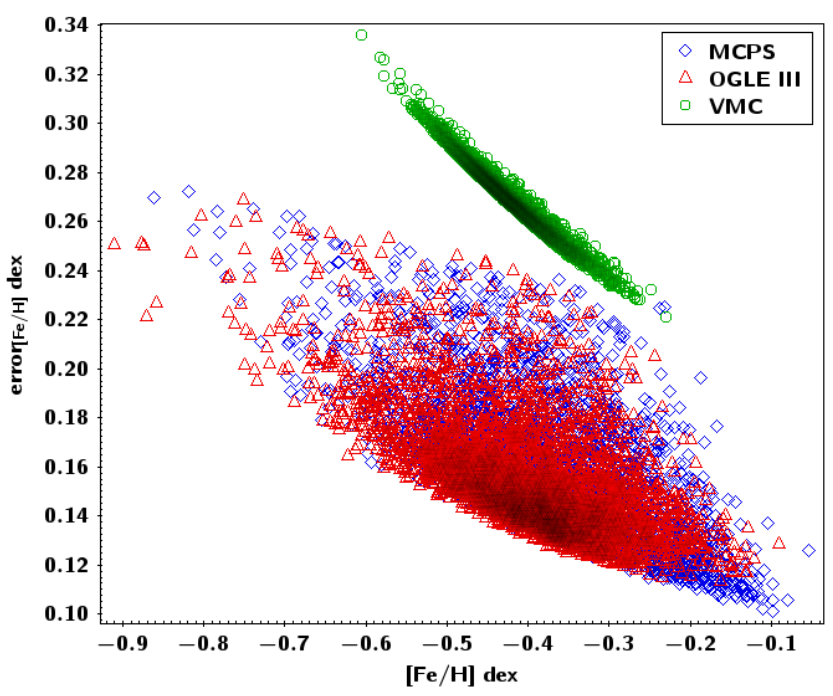

Figure 10. error $_{[\mathrm{Fe} / \mathrm{H}]}$ estimated in this study compared with $\mathrm{C} 16$ (OGLE III and MCPS).

i.e., the errors are larger than those obtained by $\mathrm{C} 16$ for the OGLE III and MCPS data sets. This is most likely related to the difference in the corresponding values of |slope| and the slope-metallicity calibration relation, which are functions of the wavelengths used in these studies. We also note a slight trend suggesting that our estimated errors are larger for metal-poor values. We find that the dispersion in the value of $\operatorname{error}_{[\mathrm{Fe} / \mathrm{H}]}$ for a given value of $[\mathrm{Fe} / \mathrm{H}]$ is smaller $(\sigma \sim 0.01 \mathrm{dex})$ for VMC compared with optical data $(\sigma \sim 0.02-0.03 \mathrm{dex})$. Referring back to the error propagation equation of $\mathrm{C} 16$, we point out that this dispersion is determined by the range of $\sigma_{\text {slope }}$ associated with the corresponding RGB |slope|.

\section{DISCUSSION}

\subsection{Impact of reddening and distance variations}

Studies in the literature show that 30 Dor and the central region of the LMC have higher reddening compared with the rest of the disc (e.g., Zaritsky et al. 2004; Haschke et al. 2011; Subramanian \& Subramaniam 2013; Górski et al. 2020; Skowron et al. 2021; Grady et al. 2021). Recent extinction maps of Mazzi et al. (2021) based on star-formation history (SFH) studies using VMC data also support these previous findings. The variation in reddening among subregions can shift the location of the RGB in the respective CMDs. Since we have adopted the technique of $\mathrm{C} 16$, this effect is accounted for in our analysis by anchoring the RGB to the densest part of the RC. However, large differential reddening variations within a subregion will broaden the RGB, leading to poorly estimated slopes. The effect of reddening is more significant in optical passbands. This is prominently observed in the metallicity maps of $\mathrm{C} 16$, where gaps are seen in the bar and in star forming regions like 30 Dor. NIR passbands offer an advantage since they suffer less from reddening compared with optical passbands. However, we still notice some gaps in our NIR metallicity maps, primarily in 30 Dor and near the bar region, which suggests that our technique fails to properly deal with subregions exhibiting large variations in reddening. Since the number of such subregions is between 3 and 6 

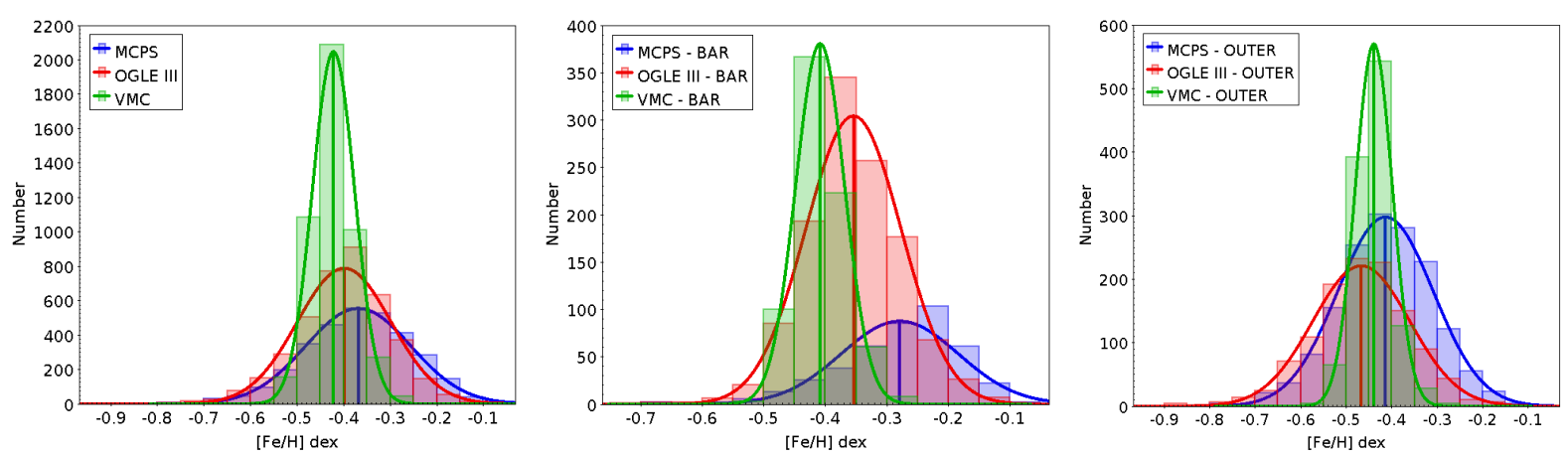

Figure 11. Comparison of the metallicity distributions across the LMC between this VMC study and those of C16 (OGLE III and MCPS) for different regions in the galaxy: left - complete coverage of each survey; middle - LMC bar; right - outer disc (see text for definitions).

per cent of our sample, their impact on our estimated mean metallicity and the radial metallicity gradient of the LMC is negligible.

In their distance modulus map Mazzi et al. (2021) show the distance variation among their adopted subregions in the LMC's disc. Our subregions are much smaller $(\sim 2-28$ times $)$ compared with Mazzi et al. (2021). Thus, it is safe to assume that there is no significant distance variation within a given subregion. By virtue of anchoring the RGB to the densest part of the $\mathrm{RC}$ for a subregion, our technique accounts for distance variations among subregions.

\subsection{Metallicity distribution within the LMC}

In Fig. 11, we show a comparison of the metallicity distributions for the complete LMC, the bar region and an outer region estimated from $\mathrm{VMC}$ data and with the results of C16. When referring to the outer LMC, we mean the regions that lie beyond a radial distance of $2.5^{\circ}$ from the LMC centre. We estimated the mean metallicity by fitting Gaussian profiles to all distributions. Overall we see that the width of the VMC distribution $(\sigma[\mathrm{Fe} / \mathrm{H}]=0.04 \mathrm{dex})$ is much smaller than the MCPS and OGLE III distributions $(\sigma[\mathrm{Fe} / \mathrm{H}]=0.10-0.11 \mathrm{dex})$ for all three specified regions in the LMC. The mean metallicity estimated using VMC data $([\mathrm{Fe} / \mathrm{H}]=-0.42 \mathrm{dex}, \sigma[\mathrm{Fe} / \mathrm{H}]=0.04 \mathrm{dex})$ is relatively metalpoor compared with MCPS $([\mathrm{Fe} / \mathrm{H}]=-0.37 \mathrm{dex}, \sigma[\mathrm{Fe} / \mathrm{H}]=$ $0.11 \mathrm{dex})$ and OGLE III data $([\mathrm{Fe} / \mathrm{H}]=-0.39 \mathrm{dex}, \sigma[\mathrm{Fe} / \mathrm{H}]=$ $0.10 \mathrm{dex})$, which peak at almost similar values. The range of metallicities estimated using VMC data is smaller compared with those obtained using MCPS and OGLE III data. Thus, we have used finer binning (0.05 dex) to construct our histograms than C16 (0.15 dex) to compare with OGLE III and MCPS results. However, note that the mean metallicity and the widths of the OGLE III and MCPS distributions are not affected by this smaller binning.

The mean metallicities of the bar region as estimated from the MCPS, OGLE III and VMC data are -0.28 dex $(\sigma[\mathrm{Fe} / \mathrm{H}]=0.09 \operatorname{dex}),-0.35 \operatorname{dex}(\sigma[\mathrm{Fe} / \mathrm{H}]=0.08 \operatorname{dex})$ and $-0.41 \operatorname{dex}(\sigma[\mathrm{Fe} / \mathrm{H}]=0.04 \mathrm{dex})$, respectively. The mean metallicity of the outer LMC is $-0.41 \operatorname{dex}(\sigma[\mathrm{Fe} / \mathrm{H}]=$ $0.10 \mathrm{dex}),-0.46 \operatorname{dex}(\sigma[\mathrm{Fe} / H]=0.11 \mathrm{dex})$ and $-0.44 \mathrm{dex}$ $(\sigma[\mathrm{Fe} / \mathrm{H}]=0.04 \mathrm{dex})$, for MCPS, OGLE III and VMC, respectively. For the MCPS and OGLE III data, the mean metallicity of the bar is prominently metal-rich as compared with the outer region. However, for the VMC data, the mean metallic- ities of the bar and outer regions are indistinguishable within the errors. The bar metallicity has lower $\sigma[\mathrm{Fe} / \mathrm{H}]$ than the outer regions, which is detectable when comparing the optical data sets. However, we cannot resolve this difference in $\sigma[\mathrm{Fe} / \mathrm{H}]$ for the VMC data set.

Even though similar passbands $(V$ and $I)$ were used for OGLE III and MCPS data, and the same calibration relation was used (after correcting for systematic effects), C16 estimated smaller values of MCPS slopes in the bar region, leading to higher values of metallicity. The central regions are affected by crowding and/or differential reddening. The MCPS data set is perhaps more affected by these issues owing to its low resolution and/or poor seeing conditions. The effect is clearly seen for common stars in the bar region in the MCPS data compared with the OGLE III and VMC data. We will discuss this in more detail in the next section. A significant fraction of bar subregions are missing in MCPS maps compared with OGLE III, implying that the bar region is poorly sampled by the MCPS. According to C16, the MCPS maps are representative of the outer LMC owing to their uniform coverage in all four directions, while the OGLE III maps represent the metallicity trend in the bar. The VMC maps provide a more comprehensive picture of both the bar and the outer regions of the LMC.

\subsection{A metallicity gradient within the LMC}

To estimate the radial MG, we consider the LMC disc to be inclined with respect to the sky plane by an angle $i$, and the position angle of the line of nodes is given by $\Theta$. We use $i=$ $25^{\circ} .7$ and $\Theta=141^{\circ} .5$ (Subramanian \& Subramaniam 2013). We assume the distance of the LMC to be $49.9 \mathrm{kpc}$ (de Grijs et al. 2014). We use these parameters in the equations of van der Marel (2001), to estimate the metallicity variation in the LMC plane. Then, we estimate a radial MG by dividing the galaxy radially into bins of $0.25 \mathrm{kpc}$ width. We also re-estimate the MGs resulting from the OGLE III and MCPS data sets (C16) out to a radius of about $4 \mathrm{kpc}$. To avoid issues owing to poor sampling, we exclude the most distant radial bins for all three data sets. In addition, for MCPS we avoid the central radial bin to avoid small-number statistics. Fig. 12 shows a comparison of the LMC MGs for all three data sets. There is an indication of decreasing metallicity with increasing distance for the VMC data. However, the variation is shallow compared with C16. Following C16, MG estimation for OGLE III is split into two parts, i.e. the 


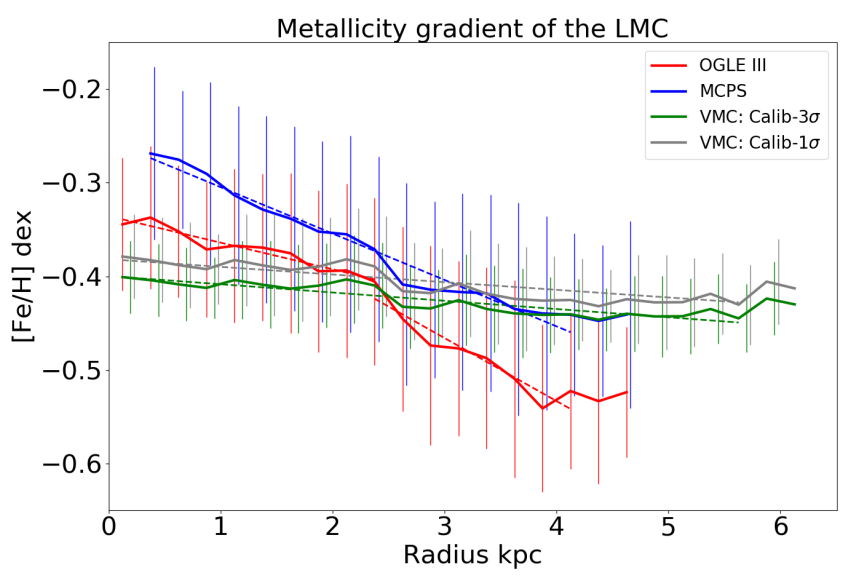

Figure 12. Radial MG estimated in NIR passbands from the VMC survey shown with respect to estimations from optical passbands (OGLE III, MCPS) from C16. The error bars denote the standard deviation about the mean. They are offset slightly along the $x$ axis for each case to avoid overlap.

central disc $(\lesssim 2.5 \mathrm{kpc}$, containing the bar) and the outer disc, $-0.028 \pm 0.003$ and $-0.057 \pm 0.009 \mathrm{dex} \mathrm{kpc}^{-1}$, respectively. The MCPS gradient is estimated at $-0.049 \pm 0.002$ dex $\mathrm{kpc}^{-1}$. The newly estimated MGs for OGLE III and MCPS are very similar to those of $\mathrm{C} 16$, although the geometric parameters assumed in their studies $\left(i=37^{\circ} .4, \Theta=141^{\circ} .2\right)$ were different. Using the VMC data we estimate a MG of $-0.008 \pm 0.001 \mathrm{dex}^{\mathrm{kpc}}{ }^{-1}$ out to a radius of $\sim 6 \mathrm{kpc}$, with a $y$ intercept of $-0.40 \pm 0.004$, and $r=0.91$. The trend estimated using the $1 \sigma$-clipped calibration relation (Equation 2) gives $\mathrm{MG}=-0.010 \pm 0.001 \mathrm{dex} \mathrm{kpc}^{-1}$. This signifies that use of either of our different slope--metallicity relations has a negligible effect on the estimated MG.

The mean metallicity per radial bin seems to be in agreement for the three data sets owing to large standard deviation about the mean. However, the differences are larger in the innermost ( 0 to $1 \mathrm{kpc}$ ) and outer $(>4-5 \mathrm{kpc}$ ) parts of the galaxy. The mean metallicity per radial bin is higher in the inner regions for MCPS, whereas OGLE III and VMC agree within the errors. At larger radii the MCPS and VMC values are similar, but OGLE III values are relatively metal-poor. We inspected this to check whether this might be owing to differences in the areas for the subregions adopted for this study and in C16. This had a negligible effect on their studies, since they were not interested in the average metallicity of each subregion, but instead they determined the global average and its variation across the LMC. Since the depth and resolution of MCPS and OGLE III data are different, C16 used different area binning criteria for both data sets. Thus, at a given location the sizes of OGLE III and MCPS subregions are different. $\mathrm{C} 16$ found that the difference between the RGB slope values (hence metallicity) at a given location for OGLE III and MCPS were well within the mutual error bounds.

To check the effect of variable areas, we selected subregions from the three data sets within the inner (0 to $1 \mathrm{kpc})$ and outer $(>4-5 \mathrm{kpc})$ LMC which had mean metallicities per radial bin similar to that observed in Fig.12. For subregions at radii from 0 to $1 \mathrm{kpc}$, we sampled subregions with metallicities between -0.250 to -0.30 dex for MCPS; -0.325

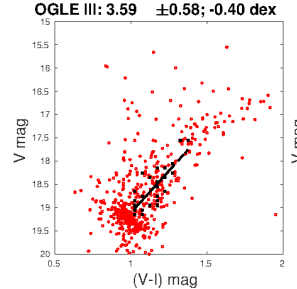

(V-I) mag

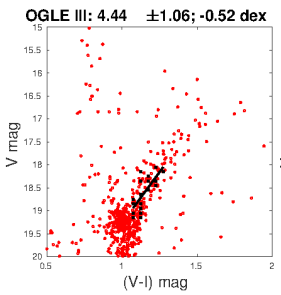

52 dex

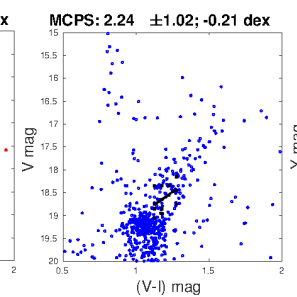

$2.24 \pm 1.02 ;-0.21$ dex

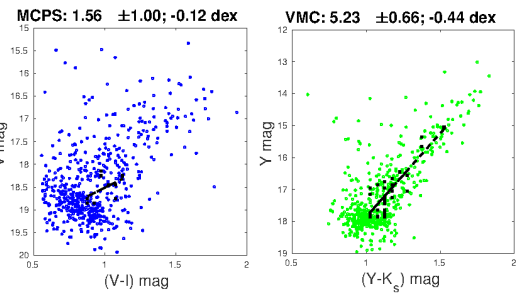

$\left(\mathrm{Y}-\mathrm{K}_{\mathrm{s}}\right) \mathrm{mag}$

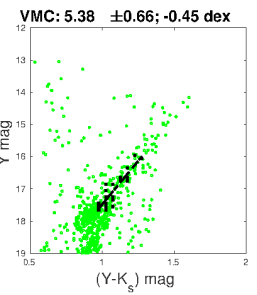

Figure 13. CMDs of common stars for OGLE III, MCPS and $\mathrm{VMC}$ data for a sample subregion, within the inner LMC (0 kpc to $1 \mathrm{kpc}$, top) and the outer LMC ( $4 \mathrm{kpc}$ to $5 \mathrm{kpc}$, bottom). The corresponding $\mathrm{RGB}$ slopes and $[\mathrm{Fe} / \mathrm{H}]$ values are listed in the panels' titles.

to -0.375 dex for OGLE III; and -0.375 to -0.425 dex for VMC. For subregions at radii from 4 to $5 \mathrm{kpc}$ we used -0.40 to -0.45 dex for MCPS; -0.50 to -0.55 for OGLE III; and -0.40 to -0.45 dex for VMC. As an example, CMDs of a subregion in the inner and outer LMC are shown in Fig. 13 for the stars in common among the three data sets. We estimated the slopes for the OGLE III and MCPS data sets following C16 and calibrated them to metallicities using their equation (1). The VMC slopes were estimated using the technique outlined in this study and calibrated by our Equation (1). We find that the differences in metallicity between the MCPS compared with OGLE III and VMC remain and are similar to what is observed in the mean metallicity per radial bin in the inner LMC. The same is noted between OGLE III compared with MCPS and VMC in the outer LMC. The CMDs also show that the MCPS data suffer from crowding issues and/or poor seeing conditions. We checked this for additional subregions. This effect is more pronounced in the central regions compared with the outer regions, leading to a broadening of RGB and a shallow RGB slope, hence metal-rich regions for MCPS. Thus, we suggest that at optical wavelengths the OGLE III results are more reliable than their MCPS counterparts. Thus, the difference in trends observed in Fig. 12 is not caused by differences in the areas covered by the subregions. This is possibly related to the systematic differences between the filter systems (optical and NIR), and the slope-metallicity calibration relation, which is a function of wavelength.

\subsubsection{Comparison with other studies}

The MG estimated by Cioni (2009) out to a distance of $8 \mathrm{kpc}$ using the $\mathrm{C} / \mathrm{M}$ ratios of the field AGB population is $-0.047 \pm 0.003 \mathrm{dex} \mathrm{kpc}{ }^{-1}$, with an $y$ intercept of $-1.04 \pm 0.01$ dex. Their radial MG is more consistent with OGLE III and MCPS than with our VMC results. This is perhaps related to the age difference ( $\sim$ a few Gyr) between AGB and RGB stars. In their fig. 2, Cioni (2009) also show the radial variation in spectroscopically derived metallicities of field RGBs 
(from Cole et al. 2005; Pompéia et al. 2008; Carrera et al. 2008) and star clusters (from Grocholski et al. 2006, 2007). This hinted at a negligible radial MG for RGBs within the inner $6 \mathrm{kpc}$ of the LMC, where the points are metal-rich compared with the AGB distribution. A few points of the spectroscopic sample lying beyond $6 \mathrm{kpc}$ were metal-poor and followed the MG trend of the AGB stars. This trend of an apparently negligible MG out to $6 \mathrm{kpc}$ agrees with our study. However, since the spectroscopic sample is statistically small compared with our photometric sample, we refrain from drawing significant conclusions from this similarity. Our results are also shallow compared with Grady et al. (2021), who estimated a MG of $-0.048 \pm 0.001 \mathrm{dex} \mathrm{kpc}^{-1}$, with a $y$ intercept of $-0.656 \pm 0.004$ dex for LMC RGB stars out to a radius of $\sim 12 \mathrm{kpc}$ (their fig. 10). These authors used machine learning techniques to combine photometric (Gaia DR2, 2MASS, WISE) and spectroscopic estimates (APOGEE). While estimating the MG these authors excluded the central regions (within $\sim 3 \mathrm{kpc}$ ) so as to avoid the metal-rich bar and focus on the LMC disc. In doing so, they mitigated the fact that their metallicity predictions for the most metal-rich stars, which dominate centrally, incurred a degree of bias $(\sim 0.1-0.2 \mathrm{dex})$ in their regression model.

\subsubsection{Asymmetric radial metallicity gradient}

To check if there is any asymmetry in our radial MG, in Fig. 14 we show the radial MG for four different directions in the LMC with respect to the optical centre. The radial trend is estimated out to $6 \mathrm{kpc}$ for the North East (NE), South East (SE) and SW quadrants, but for the NW region only out to $5 \mathrm{kpc}$. The overall trends in all four quadrants are shallow and the mean metallicity per radial bin agrees within the errors. However, their appearances are suggestive. In the eastern quadrants we see a metal-rich plateau followed by a dip in metallicity values beyond the inner $2.5-3 \mathrm{kpc}$. The plateau for the NE quadrant is more metal-rich compared with the SE and western quadrants. The trend in the SW portion, in the direction of the Bridge and the SMC, is relatively metal-poor compared with all quadrants.

We also checked the variation in metallicity with respect to the position angle (PA). Fig. 15 shows that overall all points are scattered between -0.6 dex to -0.2 dex. However, we observe more metal-poor points in the southern LMC, especially in the $\mathrm{SW}$ portion $\left(180^{\circ}\right.$ to $\left.270^{\circ}\right)$ compared with the northern regions. We estimated mean metallicities by binning along the PA and overplotted them to check for any variations. Although these values for each PA bin are in agreement within the standard deviation about the mean, they are indicative of a possible trend similar to what was found radially. We see some sharp variations in the $\mathrm{NE}\left(0^{\circ}\right.$ to $\left.90^{\circ}\right)$, $\mathrm{SE}\left(90^{\circ}\right.$ to $\left.180^{\circ}\right)$ and $\mathrm{SW}$ regions compared with the NW $\left(270^{\circ}\right.$ to $\left.360^{\circ}\right)$ region where the variation is smooth. The plot is indicative of an azimuthal MG, with more variations in the East compared with the West. This is possibly related to the effect of an non-axisymmetric bar in the LMC. Grady et al. (2021) determined the metallicity profile along directions aligned with the projected major and minor axes of the bar in their fig. 11. That figure shows a flat gradient in the centre and a negative MG with increasing distance from the central regions, with strong asymmetries. Thus, our derived

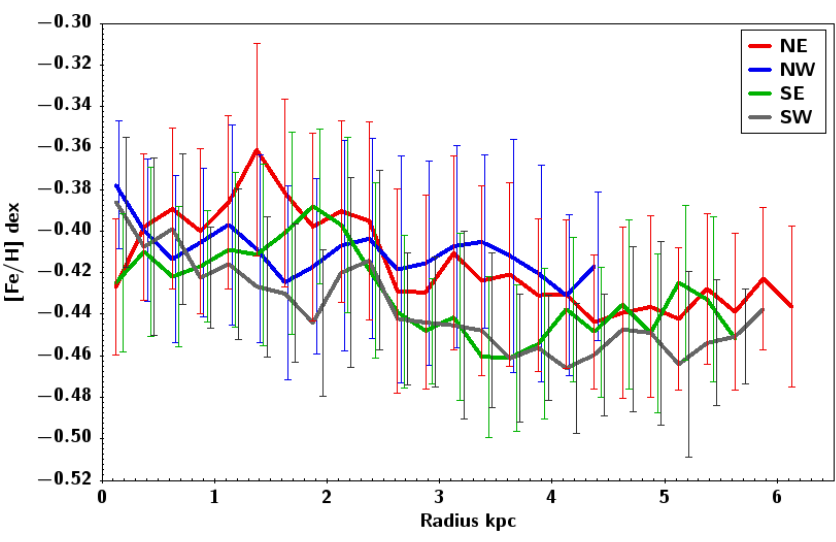

Figure 14. Radial MGs in all four directions. The errors represent the standard deviation about the mean in each radial bin. The error bars have been shifted slightly along the $x$ axis to avoid overlap.

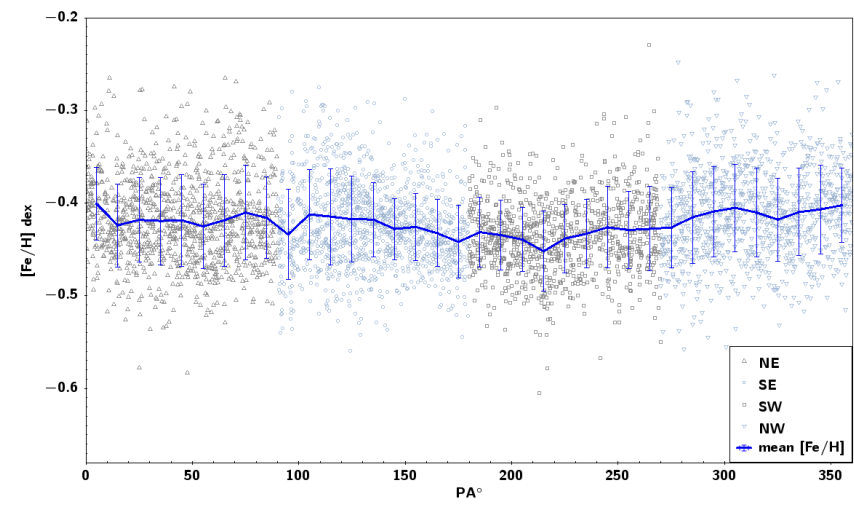

Figure 15. Metallicity distribution as a function of PA for all four directions. The mean metallicity in all PA bins are shown as blue points. The standard deviation about the mean is shown as error bars.

asymmetry in the radial MG of the LMC is consistent with Grady et al. (2021).

\subsubsection{Interpretation of metallicity gradient}

Mazzi et al. (2021) studied the SFH of the LMC using VMC data over an area of $96 \mathrm{deg}^{2}$, twice larger than Harris \& Zaritsky (2009) who used the MCPS survey data. The SFH derived by the former authors is similar to that of Harris \& Zaritsky (2009), but presents a lower star-formation rate (SFR) at young ages. Their maps show that the SFR at ages younger than $63 \mathrm{Myr}$ is patchy and centralised. At older ages $(<1.6$ Gyr), the SFR extends to larger radii. The SFR is concentrated in the bar region and in three well-defined spiral arms. As we move to ages older than $1.6 \mathrm{Gyr}$, the bar and spiral features become less prominent and eventually the SFH appears more like a circular distribution. The period of enhanced star formation in the LMC occurs approximately between 500 Myr and $4 \mathrm{Gyr}$, with two peaks of enhanced SFR: in the age intervals $630 \mathrm{Myr}$ to $1 \mathrm{Gyr}$ and 1.6-4 Gyr (where the former is associated with regions in the LMC bar). The RGB and RC star contributions to the SFH are for ages older than 1.6 Gyr. Since we are using the RGBs as our tracer, our radial MG trend is possibly representative 
of a population with ages $\geq 1-1.6$ Gyr. Mazzi et al. (2021) report no significant $\mathrm{MG}$ in the $\mathrm{LMC}$ and retrieve only a scattered distribution of metallicity that deviates from the initially assumed age-metallicity relation. The authors associate this with the relatively lower sensitivity of NIR bands to variations in metallicity compared with optical passbands. According to Mazzi et al. (2021) the dependence on metallicity is related to subtle changes in the mean RGB slope, in the position and shape of the RC, and in the mean colour (compared with the RGB) and slope of the main sequence. The negligible radial MG estimated in this study using the RGB slope possibly reflects the interpretation of Mazzi et al. (2021).

The metal-enhanced bar highlighted by this study and by previous authors (C16, Grady et al. 2021) is consistent with the findings of Fraser-McKelvie et al. (2019). These authors studied a sample of 128 barred galaxies and found that both the age and metallicity gradients are flatter in the bar as opposed to the discs of their galaxies. The presence of a bar appears to flatten or even erase abundance gradients (Alloin et al. 1981), probably by inducing non-circular motions in the gas of the disc. Our results could indicate that bars are efficient in radially mixing their stellar populations even in a dwarf, one-armed spiral galaxy like the LMC, just like it does in early- and late-type barred spiral galaxies (Seidel et al. 2016). Bekki \& Chiba (2005) investigated the evolution of the LMC's MG using chemo-dynamical simulations. Their fig. 10 shows a steep gradient out to $3 \mathrm{kpc}$ and a flattening at larger radii. Since a steep initial gradient is assumed in their simulation, the tidal interaction with the SMC and the MW, and the dynamical action of the LMC bar flattened the original MG in the model. Their steeper gradient in the central region is more consistent with the MCPS results of C16, whereas the flattened MG in the outer parts in more consistent with the present study. Our result implies that dynamical mixing of stellar populations with different metallicities by the LMC bar is perhaps more efficient than predicted by the simulations of Bekki \& Chiba (2005).

Magrini et al. (2016) found that the radial migration of stars can contribute to a flattening of the MG in the outer parts of spiral galaxies (e.g., M31, M33). The effect of stellar migration on the metallicity distribution is also observed in the MW (Loebman et al. 2016). The flattening of the MG noticed in the LMC's outer regions is possibly suggestive of a similar effect, whereby the metal-rich stellar populations from the centre migrate outwards. There could be many reason behind radial migration, e.g., transient spirals, mergers and interaction with satellite galaxies, or interactions between bars and spiral arms. The actual reason behind this phenomenon in the LMC needs further verification, which is beyond the scope of this work. The radially asymmetric MG in the LMC suggests a relatively flat trend on the western side, in the direction of the Bridge and the SMC, compared with the eastern side. The SW portion of the LMC's disc is known to be warped (by $\sim 4 \mathrm{kpc}$; Choi et al. 2018) in the direction of the SMC because of their interaction history. The relatively metal-poor metallicity trend in the SW LMC hints at population mixing in this region, similar to the eastern SMC (C20), stemming from the LMC and SMC's tidal interaction.

\section{SUMMARY}

We have successfully extended our technique of combining large-scale photometric and spectroscopic data to estimate NIR metallicity maps of the LMC. The results can be summarised as follows:

(i) Our NIR metallicity maps exceed the previously obtained metallicity maps of C16 in terms of area coverage (three times larger), revealing trends across $105 \mathrm{deg}^{2}$ of the LMC. The spatial resolution of the NIR maps ranges between $50.37 \times 69.68 \mathrm{pc}^{2}$ and $201.79 \times 278.73 \mathrm{pc}^{2}$ for the innermost and outermost subregions, respectively.

(ii) We estimated RGB slopes in the $Y$ versus $Y-K_{\mathrm{s}} \mathrm{CMD}$ of $\sim 4663$ subregions within a radius of $6 \mathrm{kpc}$ and converted the slopes to metallicity values using spectroscopic data of field RGs.

(iii) The mean metallicity of the LMC based on VMC data is $-0.42 \pm 0.04 \mathrm{dex}$ out to a radius of $6 \mathrm{kpc}$. This agrees well with the mean metallicities estimated in previous photometric and spectroscopic studies of RGs.

(iv) The RGB population drawn from a spatially homogeneous large-area photometric data set shows the existence of a negligible MG $\left(-0.008 \pm 0.001 \mathrm{dex} \mathrm{kpc}{ }^{-1}\right)$ within $6 \mathrm{kpc}$. The estimated gradient is shallow compared with previous largearea photometric MG estimates (C16, Cioni 2009; Grady et al. 2021). Our NIR metallicity maps are possibly tracing a stellar population that is older than 1-1.6 Gyr.

(v) Our results indicate that the LMC bar has played a role in turning the central region metal-rich and flattening the gradient within the central $3 \mathrm{kpc}$. The trend of a flattened gradient in the outer LMC is possibly caused by mixing induced by the LMC bar or by radial migration of the metal-rich population from the centre outwards.

(vi) The LMC has an asymmetric radial MG, possibly caused by the non-axisymmetric bar. The eastern disc has a steeper metallicity trend compared with the western disc. The metal-poor and flattened trend in the SW, in the direction of the Bridge and the SMC, is possibly analogous to a similar trend noted in the eastern SMC by C20. We suspect that this could be a result of the tidal interaction in the LMC-SMC system.

(vii) Our study supports further spectroscopic investigations of the LMC using large-scale multi-fibre spectrographs like the $4 \mathrm{~m}$ Multi-Object Spectroscopic Telescope (4MOST; de Jong et al. 2014, 2019). Detailed studies of individual chemical abundances and chemo-kinematics for regions like the SW quadrant of the LMC and the eastern part of the SMC may yield important clues as to the LMC-SMC interaction history.

\section{ACKNOWLEDGEMENTS}

We thank the Cambridge Astronomy Survey Unit (CASU) and the Wide Field Astronomy Unit (WFAU) in Edinburgh for providing the necessary data products under the support of the Science and Technology Facilities Council (STFC) in the UK. This study was based on observations made with VISTA at the La Silla Paranal Observatory under programme ID 179.B-2003. This project has received funding from the European Research Council (ERC) under the European Union's Horizon 2020 research and innovation pro- 
gramme (grant agreement 682115). We acknowledge Stefano Rubele for generating the PSF photometry catalogue used in this work. We are grateful for the use of the computer cluster at the University of Hertfordshire, to Simone Zaggia for making available local computers at the University of Padova, and acknowledge the ERC Consolidator Grant funding scheme (project STARKEY, grant agreement 615604) for supporting S. Rubele's work. We thank Andrew A. Cole for providing CaT spectroscopic data of field RGs in the LMC which helped in estimating the slope-metallicity calibration relation. We also thank Martin A. T. Groenewegen for his comments which helped improve the manuscript. We thank the anonymous referee for their suggestions which helped improve the clarity of the manuscript.

\section{DATA AVAILABILITY}

The PSF catalogue of the LMC used in our work is proprietary to the VMC team, with a data release planned in mid 2022 .

\section{REFERENCES}

Alloin D., Edmunds M. G., Lindblad P. O., Pagel B. E. J., 1981, A\&A, 101, 377

Bekki K., Chiba M., 2005, MNRAS, 356, 680

Besla G., Kallivayalil N., Hernquist L., van der Marel R. P., Cox T. J., Kereš D., 2012, MNRAS, 421, 2109

Carrera R., Gallart C., Hardy E., Aparicio A., Zinn R., 2008, AJ, 135,836

Choi Y., et al., 2018, ApJ, 866, 90

Choudhury S., Subramaniam A., Cole A. A., 2016, MNRAS, 455, 1855

Choudhury S., et al., 2020, MNRAS, 497, 3746

Cioni M.-R. L., 2009, A\&A, 506, 1137

Cioni M. R. L., et al., 2011, A\&A, 527, A116

Cole A. A., Tolstoy E., Gallagher III J. S., Smecker-Hane T. A., 2005, AJ, 129, 1465

Dalton G. B., et al., 2006, Proc. SPIE, 6269, 62690X

Diaz J., Bekki K., 2011, MNRAS, 413, 2015

El Youssoufi D., et al., 2019, Monthly Notices of the Royal Astronomical Society, 490, 1076-1093

El Youssoufi D., et al., 2021, MNRAS,

Feast M. W., Abedigamba O. P., Whitelock P. A., 2010, MNRAS, 408, L76

Fraser-McKelvie A., et al., 2019, MNRAS, 488, L6

Fujimoto M., Murai T., 1984, in van den Bergh S., de Boer K. S. D., eds, IAU Symposium Vol. 108, Structure and Evolution of the Magellanic Clouds. pp 115-123, doi:10.1017/S0074180900040080

Gaia Collaboration et al., 2021, A\&A, 649, A7

Gardiner L. T., Sawa T., Fujimoto M., 1994, MNRAS, 266, 567

González-Fernández C., et al., 2018, MNRAS, 474, 5459

Górski M., et al., 2020, ApJ, 889, 179

Grady J., Belokurov V., Evans N. W., 2021, ApJ, 909, 150

Grocholski A. J., Cole A. A., Sarajedini A., Geisler D., Smith V. V., 2006, AJ, 132, 1630

Grocholski A. J., Sarajedini A., Olsen K. A. G., Tiede G. P., Mancone C. L., 2007, AJ, 134, 680

Harris J., Zaritsky D., 2009, AJ, 138, 1243

Haschke R., Grebel E. K., Duffau S., 2011, AJ, 141, 158

Kallivayalil N., van der Marel R. P., Alcock C., Axelrod T., Cook K. H., Drake A. J., Geha M., 2006a, ApJ, 638, 772
Kallivayalil N., van der Marel R. P., Alcock C., 2006b, ApJ, 652, 1213

Kallivayalil N., van der Marel R. P., Besla G., Anderson J., Alcock C., 2013, ApJ, 764, 161

Lapenna E., Mucciarelli A., Origlia L., Ferraro F. R., 2012, ApJ, 761,33

Loebman S. R., Debattista V. P., Nidever D. L., Hayden M. R., Holtzman J. A., Clarke A. J., Roškar R., Valluri M., 2016, ApJ, 818, L6

Mackey A. D., Koposov S. E., Erkal D., Belokurov V., Da Costa G. S., Gómez F. A., 2016, MNRAS, 459, 239

Mackey D., Koposov S., Da Costa G., Belokurov V., Erkal D., Kuzma P., 2018, ApJ, 858, L21

Magrini L., Coccato L., Stanghellini L., Casasola V., Galli D., 2016, A\&A, 588, A91

Majewski S. R., et al., 2017, AJ, 154, 94

Mazzi A., et al., in press, 2021, MNRAS

Murai T., Fujimoto M., 1980, PASJ, 32, 581

Nidever D. L., Monachesi A., Bell E. F., Majewski S. R., Muñoz R. R., Beaton R. L., 2013, ApJ, 779, 145

Nidever D. L., et al., 2020, ApJ, 895, 88

Niederhofer F., et al., 2021, MNRAS, 502, 2859

Olsen K. A. G., Zaritsky D., Blum R. D., Boyer M. L., Gordon K. D., 2011, ApJ, 737, 29

Olszewski E. W., Schommer R. A., Suntzeff N. B., Harris H. C., 1991, AJ, 101, 515

Pompéia L., et al., 2008, A\&A, 480, 379

Rubele S., et al., 2012, A\&A, 537, A106

Rubele S., et al., 2015, MNRAS, 449, 639

Seidel M. K., Falcón-Barroso J., Martínez-Valpuesta I., SánchezBlázquez P., Pérez I., Peletier R., Vazdekis A., 2016, MNRAS, 460,3784

Skowron D. M., et al., 2021, ApJS, 252, 23

Skrutskie M. F., et al., 2006, AJ, 131, 1163

Subramanian S., Subramaniam A., 2010, A\&A, 520, A24

Subramanian S., Subramaniam A., 2013, A\&A, 552, A144

Subramanian S., et al., 2017, MNRAS, 467, 2980

Sutherland W., et al., 2015, A\&A, 575, A25

Tanaka K. I., 1981, PASJ, 33, 247

Udalski A., et al., 2008, Acta Astron., 58, 89

Wang J., Hammer F., Yang Y., Ripepi V., Cioni M.-R. L., Puech M., Flores H., 2019, MNRAS, 486, 5907

Westerlund B. E., 1997, The Magellanic Clouds

Wright E. L., et al., 2010, AJ, 140, 1868

Zaritsky D., Harris J., Thompson I. B., Grebel E. K., 2004, AJ, 128,1606

de Grijs R., Bono G., 2015, AJ, 149, 179

de Grijs R., Wicker J. E., Bono G., 2014, AJ, 147, 122

de Jong R. S., et al., 2014, in Ramsay S. K., McLean I. S., Takami H., eds, Society of Photo-Optical Instrumentation Engineers (SPIE) Conference Series Vol. 9147, Ground-based and Airborne Instrumentation for Astronomy V. p. 91470M, doi: $10.1117 / 12.2055826$

de Jong R. S., et al., 2019, The Messenger, 175, 3

de Vaucouleurs G., Freeman K. C., 1972, Vistas in Astronomy, 14, 163

van der Marel R. P., 2001, AJ, 122, 1827

This paper has been typeset from a $\mathrm{T}_{\mathrm{E}} \mathrm{X} / \mathrm{LAT}_{\mathrm{E} X}$ file prepared by the author. 